\title{
Three-dimensional particle size determination in a laminar diffusion flame by tomographic laser-induced incandescence
}

\author{
Florian J. Bauer ${ }^{1,2,3} \cdot$ Tao Yu $^{4} \cdot$ Weiwei Cai ${ }^{2,4} \cdot$ Franz J. T. Huber ${ }^{1,2,3} \cdot$ Stefan Will ${ }^{1,2,3}$
}

Received: 30 June 2020 / Accepted: 5 December 2020 / Published online: 23 December 2020

(c) The Author(s) 2020

\begin{abstract}
Non-intrusive measurement techniques are required to gain a comprehensive understanding about the processes of soot formation, growth and oxidation. Time-resolved laser-induced incandescence (TiRe-LII), commonly performed 0D or 2D within a flame, has proven to be a very suitable tool for the in situ sizing of soot primary particles. In this work, the technique is expanded to the third dimension by employing volumetric illumination and coupling it with a tomographic approach, which allows to computationally gain 3D information from 2D images taken at various angles. To minimize experimental cost, an approach using nine fiber bundles arranged in a semicircle around the flame and imaging the light onto a single camera is used. The technique is demonstrated on an ethene diffusion flame on a standard burner, providing spatially resolved 3D particle sizes. One focus of this work is to reveal the influence of input parameters such as the local bath gas temperature, which we measured by two-color pyrometry, and local laser fluence, which are both required for an accurate evaluation of the local particle size. It is shown that the assumption of an average temperature may result in a wrong picture even of qualitative soot size evaluation. In the end, a concept is proposed for a simultaneous determination of the 3D distribution of particle sizes through TiRe-LII and the required bath gas temperature via two-color pyrometry using a tomographic approach with only three cameras.
\end{abstract}

\section{Introduction}

In fuel rich combustion zones soot nanoparticles form from polycyclic aromatic hydrocarbon (PAH) clusters [1]. In a second step the individual primary particles form aggregates through collision, accompanied by ongoing surface growth and coagulation of PAHs and primary particles as well as primary particles among each other [2]. For a comprehensive

Stefan Will

Stefan.Will@fau.de

1 Lehrstuhl für Technische Thermodynamik (LTT), FriedrichAlexander-Universität Erlangen-Nürnberg (FAU), Am Weichselgarten 8, 91058 Erlangen, Germany

2 Erlangen Graduate School in Advanced Optical Technologies (SAOT), Friedrich-Alexander-Universität Erlangen-Nürnberg (FAU), Paul-Gordan-Straße 6, 91052 Erlangen, Germany

3 Cluster of Excellence Engineering of Advanced Materials (EAM), Friedrich-Alexander-Universität Erlangen-Nürnberg (FAU), Nägelsbachstraße 49b, 91052 Erlangen, Germany

4 Key Laboratory of Education Ministry for Power Machinery and Engineering, School of Mechanical Engineering, Shanghai Jiao Tong University, 800 Dongchuan Road, Shanghai 200240, China understanding of this formation and growth process as well as of oxidation in the following step, the determination of the size of these primary particles is of major interest. There are various measurement techniques to investigate soot formation during combustion. While some of them are based on sampling procedures, in situ techniques allow to gain insight without perturbing the system under investigation [3]. Optical metrology plays an outstanding role in this context.

For years, time-resolved laser-induced incandescence (TiRe-LII) has been used to determine the size of nanoparticles, particularly of soot [4]. LII is based on heating an ensemble of soot particles with a short laser pulse up to approximately $3000-4000 \mathrm{~K}$ and detecting and analyzing the thermal radiation signal during the subsequent cooling to bath gas temperature.

Because of their larger surface-to-volume ratio small particles show a faster signal decay as compared to larger ones. A regression between the measurement data and simulated signals from sophisticated cooling models allows to infer the particle size from the LII signal decay. To model the cooling accurately, ambient conditions, such as the bath gas temperature and local fluence of the laser radiation have to be known precisely $[5,6]$. Even small uncertainties in those ambient 
parameters can lead to large deviations and uncertainties in the determined particle size, a result of the ill-posedness of the problem [7, 8]. Therefore, accurate measurements of the absolute particle size distribution might be challenging, while the determination of relative changes in the particle sizes within certain regions in a semi-quantitative way are very beneficial, especially for large measurement volumes. From the peak incandescence signal the soot volume fraction $f_{V}$ can additionally be determined [9].

While originally TiRe-LII was mainly used for pointwise measurements [10], Will et al. [11] first realized 2D measurements, expanding the capabilities of the technique tremendously. Sun et al. [12] presented an approach to apply the planar technique in turbulent flames, which is relevant for many practical applications [13]. The challenge of 2D techniques compared to a pointwise detection with photomultiplier tubes derives from the fact that cameras have a limited repetition rate to record the signal decay curve. Therefore, approaches have been presented where either a shifting of the camera gating time [14] or an ultra-highspeed camera, capable of capturing several images within a few hundred nanoseconds [15] were used. While latter techniques allow to record the signal decay curve after a single laser pulse of one spatial particle size distribution in a turbulent process, they do not necessarily provide insight in an ongoing growth process, which usually takes place on a millisecond time scale and below [16]. Therefore, a laser with a high repetition rate and multiple high-speed cameras that can be operated in the $\mathrm{kHz}$ range are required. Yet, for complex realistic combustion systems, 3D information about the particle size and volume fraction evolution is necessary for a better understanding of the combustion process. For 3D measurements of soot volume fraction Hult et al. [17] used LII and scanned the flame layer by layer using a rapid mirror, but they did not measure particle sizes. Meyer et al. [18] presented a tomographic set-up using seven high-speed cameras under various angles to record 3D sequences of the soot volume fraction in a turbulent flame. Yet, besides the high expense of the laboratory set-up, they did not measure primary particle sizes with their approach. Recently, Hall et al. [19] presented an approach of tomographic particle sizing by TiRe-LII using three ultra-high-speed (10 MHz) cameras. Besides the cost-expensive experimental setup, the approach also suffered from the assumption of a constant bath gas temperature for particle size evaluation.

Set-ups employing fiber bundles were used for chemiluminescence measurements by Anikin et al. [20], however, they only performed a tomographic $2 \mathrm{D}$ reconstruction. More recent approaches enable three dimensional measurements with a few cameras capturing more projections utilizing fiber bundles $[21,22]$. In the present work, we coupled TiReLII with a tomographic approach to determine 3D primary particle sizes in a laminar diffusion flame. To that end, we used an approach employing nine customized fiber bundles arranged in a semicircle around the flame and imaging the light onto a single camera, which avoids the need for many cameras, yet at the cost of the image resolution. To our knowledge, this is the first demonstration of 3D-TiRe-LII for primary particle sizing employing the evaluations for 3D temperatures and laser profiles. As local bath gas temperature is a key parameter for accurate particle sizing, in the end we suggest an approach how the present work can be extended by a simultaneous tomographic measurement by two-color pyrometry, which is performed sequentially in this work.

\section{Theoretical background}

\subsection{LII model}

There are basically two ways to evaluate TiRe-LII signals: two-color LII is based on the detection of the LII signal at two different wavelengths, from which the particle temperature can be computed, allowing for a direct measurement and comparison of the cooling curve with a simulated one [23]. For single-color LII the signal decay curve is recorded at only one detection wavelength and then compared with a simulated one from the heat transfer model. Prospecting for turbulent measurements and the need of high-speed detection equipment, the latter method is preferred in this work, reducing the experimental expenditure by a factor of two. While the accuracy is somewhat reduced (due to optical and heat transfer parameters remaining in the simulation of the signal curve) [24], the single-color approach is well suited to describe how soot size evolves within a flame. The heat transfer model used to determine the primary particle size is described in detail in previous work [7]. The measurement model presented therein is based on polydisperse size distributions for the primary particles diameter $d_{\mathrm{p}}$ as well as the radius of gyration of the aggregates $R_{\mathrm{g}}$. In this work, however, we simplify the model by first assuming a monodisperse primary particle size. Furthermore, we use an effective accommodation coefficient $\alpha_{\text {eff }}=0.2$ [25] to account for the shielding effect arising from the aggregate structure. The value of $\alpha$ is known to vary with chemical (e.g., soot age or surface chemistry) as well as physical (e.g., the aggregate morphology) properties of soot and the local gas composition [26-28]. Anyhow, it is common practice to assume a constant value of $\alpha$ if no further knowledge is available about its local distribution in the investigated flame. Beside the thermal accommodation coefficient other parameters such as $E(\tilde{m})$ are known to vary with soot maturity as described in detail by Olofsson et al. in one-dimensional premixed flames [29]. Overall, there is a wide span of reported values in the literature [30], and because of lack of knowledge 
of the variation of $E(\tilde{m})$ in this type of flame, this value is assumed as constant throughout the flame in this work.

The LII signal $S_{\lambda}$ at a certain detection wavelength $\lambda$ is a function of the primary particle's temperature $T_{\mathrm{p}}$ and can be described by:

$S_{\lambda}(t)=C_{\mathrm{det}} \cdot C_{\mathrm{abs}, \lambda}^{\mathrm{pp}}\left(d_{\mathrm{p}}\right) \cdot I_{\mathrm{b}, \lambda}\left(T_{\mathrm{p}}\right)$

Here, $C_{\text {det }}$ is a scaling constant accounting for the efficiency of the detection system, $C_{\mathrm{abs}, \lambda}^{\mathrm{pp}}\left(d_{\mathrm{p}}\right)$ is the absorption cross section of a primary particle under the Rayleigh approximation $d_{\mathrm{p}} \ll \lambda$, and $I_{\mathrm{b}, \lambda}$ is the blackbody spectral intensity for a specific temperature $T_{\mathrm{p}}$. The particle temperature derives from the simplified mass- and energy balance:

$\rho \cdot c_{\mathrm{p}} \cdot \frac{\pi d_{\mathrm{p}}^{3}}{6} \frac{\mathrm{d} T_{\mathrm{p}}}{\mathrm{d} t}=\dot{Q}_{\mathrm{abs}}+\dot{Q}_{\text {cond }}+\dot{Q}_{\text {subl }}$

with density $\rho=1860 \mathrm{~kg} / \mathrm{m}^{3}[31]$ and heat capacity $c_{\mathrm{p}}(T)$ [32]. The terms $\dot{Q}_{\text {abs }}, \dot{Q}_{\text {cond }}$ and $\dot{Q}_{\text {subl }}$ are the rates of energy transfer through absorption, heat conduction and sublimation, respectively, possible additional mechanisms that only have a small share in balance such as phototoemission are neglected. One feature of the model described in [7] is the coupling with a Bayesian approach. It allows to treat certain parameters of the model, e.g., the bath gas temperature, as stochastic variables rather than deterministic values. As measurement techniques deliver results afflicted with a certain error, these need to be considered for a sophisticated data evaluation: the Bayesian method is capable to account for these uncertainties in form of the prior knowledge used. In this work, we assume normally distributed probability density functions with a mean value and a standard deviation.

\subsection{Two-color pyrometry}

One key information required for reliable TiRe-LII evaluation is the local bath gas temperature $T_{\mathrm{g}}$ varying significantly within a flame. To determine local temperatures, tomographic two-color pyrometry $(2 \mathrm{CP})$ measurements are conducted using two wavelength bands, centered at $600 \mathrm{~nm}$ and $750 \mathrm{~nm}$ with a full width at half maximum (FWHM) of $50 \mathrm{~nm}$ each. The approach is described in detail elsewhere [33], the main features are summarized here. 2CP is based on Planck's law, describing the incandescence signal of a black body as a function of temperature $T$ and wavelength $\lambda$

$I_{\mathrm{b}, \lambda}(T)=\frac{2 \pi h c^{2}}{\lambda^{5}} \frac{1}{\mathrm{e}^{\left(h c /\left(\lambda T k_{\mathrm{B}}\right)\right)}-1}$

where $h$ is Planck's constant, $c$ the speed of light and $k_{\mathrm{B}}$ Boltzmann's constant. For soot as a blue emitter [6], a correction with respect to its optical properties is required. The emissivity $\varepsilon_{\lambda}$ reduces the spectral exitance from a black body within the Rayleigh approximation $\left(d_{\mathrm{p}} \ll \lambda\right)$ according to

$I_{\lambda}(T)=\varepsilon_{\lambda} \cdot I_{\mathrm{b}, \lambda}(T)=\frac{4 \pi d_{\mathrm{p}} E(\tilde{m})}{\lambda} \cdot I_{\mathrm{b}, \lambda}(T)$

Here, $E(\tilde{m})$ is the absorption function, which varies with wavelength. For the description of the wavelength-dependence we rely on the carefully performed work by Yon et al. [34]. Based on their work, we use values of $E(\tilde{m})=0.28$ at $750 \mathrm{~nm}$ and $E(\tilde{m})=0.30$ at $600 \mathrm{~nm}$ for temperature determination, the latter one also being used in the LII model described above. The ratio of two signals at different wavelengths $I_{\mathrm{r}}$ is therefore a unique function of temperature,

$I_{\mathrm{r}}=C_{\mathrm{cal}} \frac{\varepsilon_{\lambda_{1}} \cdot I_{\mathrm{b}, \lambda_{1}}(T)}{\varepsilon_{\lambda_{2}} \cdot I_{\mathrm{b}, \lambda_{2}}(T)}$

where $C_{\text {cal }}$ is the calibration constant obtained using a well-defined radiation source, as described in the Sect. 3.

\subsection{Tomographic reconstruction}

A tomographic reconstruction uses multiple 2D projections to rebuild a 3D object. Considering the forward problem, for any detection angle a 3D object can be projected onto a 2D image. Given multiple measured images from different detection angles, tomographic reconstruction requires to solve the inverse problem given by a set of equations:

$p_{s, t}=\sum_{n=1}^{N} f_{n} \cdot W_{n}(s, t)$

Here, $p_{\mathrm{s}, \mathrm{t}}$ is the intensity captured by the $s$ th pixel of the $t$-th projection, $W_{n}(s, t)$ is a weight coefficient corresponding to the $n$-th voxel and the $s$-th pixel of the $t$-th projection. The weight coefficients are determined based on the intrinsic and extrinsic parameters of the imaging and detection system, which are obtained by the MATLAB Calibration Toolbox [35]. Over the last years, multiple approaches have been presented using tomographic reconstruction in combustion diagnostics [36-38]. Due to the ill-posedness of the inversion, the quality of the reconstruction and the amplification of initial measurement noise can differ remarkably, especially with respect to the number of projection angles and their arrangement $[36,39,40]$. Furthermore, the exact reconstruction algorithm plays an important role. In our work, the algebraic reconstruction technique (ART), which is commonly used in emission tomography, is applied as it can better recover a continuous object shape, while multiplicative ART (MART) is favored in tomographic particle 
image velocimetry (PIV) for the determination of peak signals of particles [41]. We therefore decided to use the ART method, as for a first approach we wanted to make sure to have a stable reconstruction of the flame signals. However, as mentioned by Subbarao et al. [42], MART is less sensitive to noise in the input data and therefore a comparison between the reconstruction algorithms should be addressed in future work. In the ART implementation of this work, a total number of 50 iterations was used as the stop criterion. This criterion has been proven to be stable in compliance with the parameter settings as used in this work, such as the size of tomographic volume and voxel discretization. A detailed description of the reconstruction procedure is given by Floyd et al. [43].

\section{Experimental set-up}

The experimental set-up is depicted in Fig. 1. A $10 \mathrm{~Hz}$ pulsed Nd:YAG laser (Quantel Q-smart 850) with a maximum output power of $850 \mathrm{~mJ}$ at a wavelength of $1064 \mathrm{~nm}$ is used to excite the LII-signal in the probe volume. The pulse duration is $6 \mathrm{~ns}$ with an approximately Gaussian shaped temporal profile. A combination of a spherical concave and two cylindrical plano-convex lenses is used to expand the initial output beam diameter of $6 \mathrm{~mm}$ to an ellipsoidally shaped beam with a height of $\sim 30 \mathrm{~mm}$ and a width of $\sim 18 \mathrm{~mm}$ as depicted in Fig. 2b. The laser is operated at $580 \mathrm{~mJ}$ output energy per pulse, measured by a power meter, resulting in an average fluence of $0.082 \mathrm{~J} / \mathrm{cm}^{2}$, with relative shot-to-shot fluctuations of $0.6 \%$ as depicted in the histogram of 2000 single shots in Fig. 2a. Beside the overall intensity fluctuations, the beam profile shows a weak interference pattern, requiring a detailed profile measurement to account for these local fluence variations in the LII model. The average profile of eight single shots is shown in Fig. 2b, with the summation curves over both directions (black lines) overlaying the summation curves of the individual single shots in color. From shot to shot the interference pattern is maintained, the modulation of the average fluence is in the range of $3-4 \%$; this already includes the overall intensity fluctuations of $0.6 \%$.

To account for laser beam attenuation across the flame for the LII data evaluation the laser attenuation in the center position at $\mathrm{HAB}=30 \mathrm{~mm}$ was determined by extinction measurements using a broadband light source (Energetiq, EQ-99XFC LDLS) in the wavelength band from 800 to $990 \mathrm{~nm}$ and a spectrometer (Ocean Optics QEPro). With wavelength dependent $E(\tilde{m})$ values presented by Yon et al. [34] and a length $L=4.6 \mathrm{~mm}$ (flame diameter), this results in

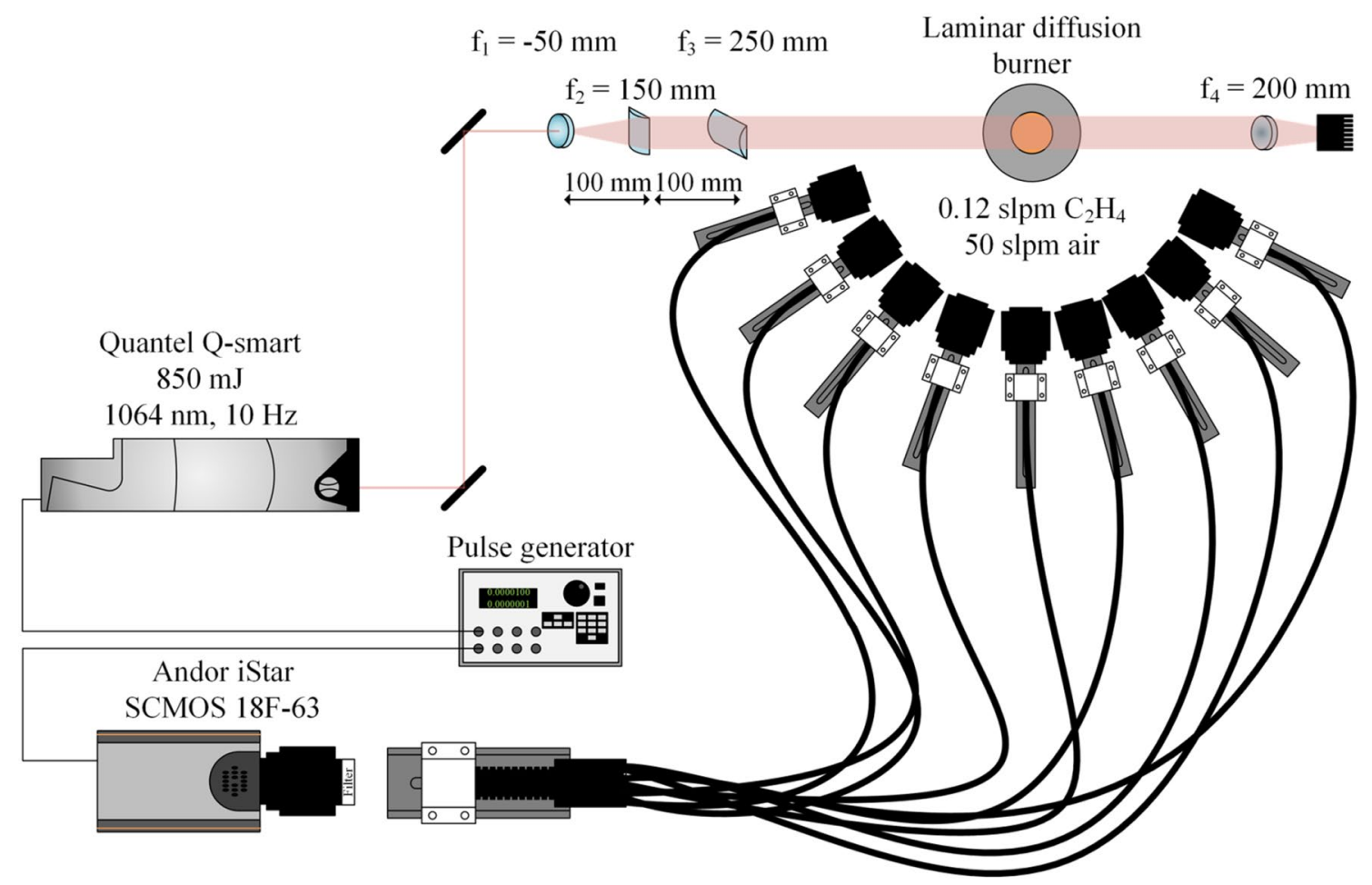

Fig. 1 Experimental set-up: An $850 \mathrm{~mJ}$ laser at $1064 \mathrm{~nm}$ is shaped to a beam giving volumetric illumination and sent through an ethene diffusion flame, which is surrounded by nine inputs of a customized fiber bundle ending in a $3 \times 3$ sub-image output, placed in front of an intensified SCMOS camera, synchronized with the laser pulse 


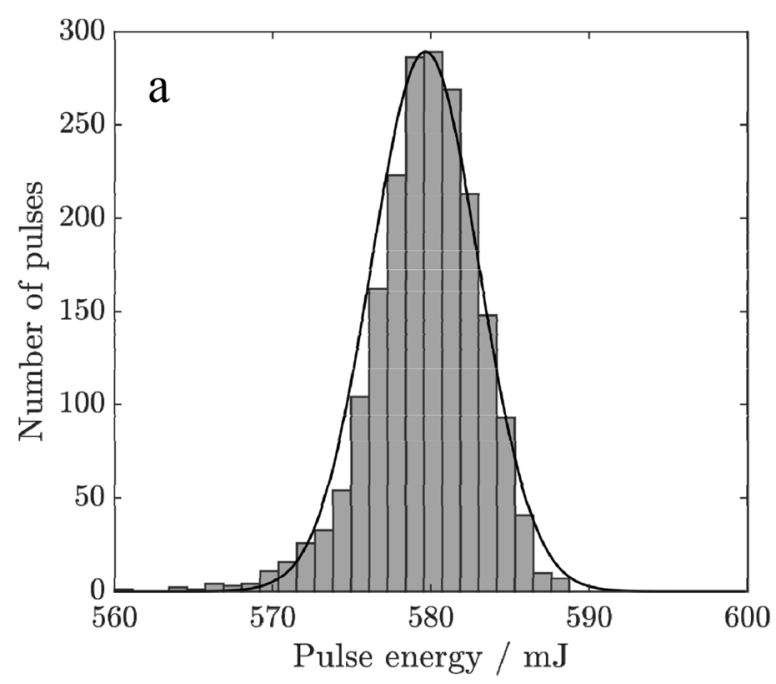

Fig. 2 a Histogram of the energy of 2000 laser pulses recorded with a power meter. The curve shows a normal distribution with a standard deviation of $0.6 \%$ of the mean value of $580 \mathrm{~mJ}$. b Average beam pro-

a mean soot volume fraction of $3.3 \mathrm{ppm}$ within the Rayleigh approximation:

$f_{V}=-\frac{\lambda \ln \left(\frac{I}{I_{0}}\right)}{6 \pi E(\tilde{m}) L}$

As the soot aggregate size was not measured in this work, the scattering contribution to the laser attenuation could not be exactly quantified. Based on the work of Sorensen [44] the scattering albedo for the particle size obtained in our work is estimated to be below $15 \%$, which could result in an overestimation of $f_{V}$ below $15 \%$. Using the extinction data, the reconstructed LII peak signals can be used to determine local $f_{V}$-values, from which the beam attenuation across the flame can be determined. Ultimately, this determination should be realized in an iterative way as the attenuation already affects the reconstructed LII signal. Yet, for this first approach we use only one pass as this error may be assumed as small.

To account for all of these fluctuations as well as the nominal sensor measurement uncertainty of $5 \%$, the local fluence $H$ was treated as a stochastic variable in the evaluation with a normally shaped probability density function and a somewhat increased standard deviation of $7 \%$ of the mean value at the respective voxel.

The laminar diffusion flame burner used in this work, described in detail by Snelling et al. [45], consists of an inner tube with an inner diameter of $D=10.9 \mathrm{~mm}$ surrounded by another tube with an outer diameter of $100 \mathrm{~mm}$ for a shroud flow of air, which is homogenized by a fill of glass spheres

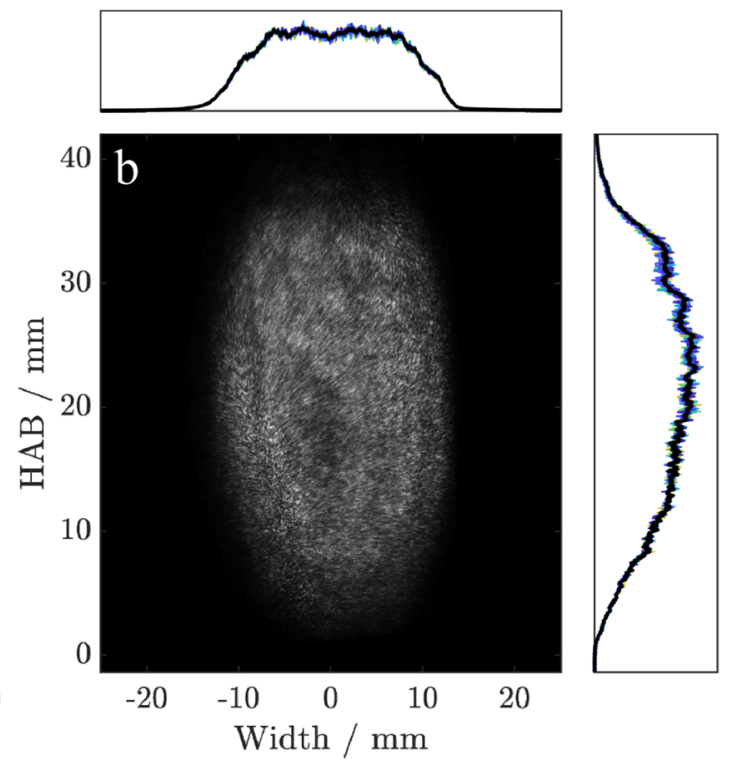

file of eight laser pulses recorded by a profile camera. On top and on the right side the summation curves in both directions of the single shots are given in color, those of the averages in black

and by two porous metal disks. For ethene a flowrate of $0.12 \mathrm{slpm}$ (standard liters per minute), for air a flowrate of $50 \mathrm{slpm}$ is adjusted resulting in a stable flame with a height of approximately $45 \mathrm{~mm}$. Compared to the flame configuration presented by Schulz et al. [46] (with a primary particle size of approximately $29 \mathrm{~nm}$ at $42 \mathrm{~mm}$ HAB center position), the reduced flow rates were chosen for two reasons. First, we wanted to capture as much of an entire flame as possible to reveal the changes in particle size, which is limited by the volumetric illumination and a sufficient laser fluence. Further, in our laboratory we had stability problems for higher flow rates because of interference from lab ventilation system. Unfortunately, for this flame no quantitative reference data are available in the literature. However, the 3D distribution of particle size will be compared qualitatively with other publications in the upcoming section.

The flame is surrounded by nine objective lenses (type: AF Nikon f/1.8) with a focal length of $50 \mathrm{~mm}$, each imaging an area of $(30 \mathrm{~mm})^{2}$ onto one of the nine fiber bundle inputs. Each input is an array of $340 \times 340$ fibers with a size of $\sim 5.8 \mathrm{~mm} \times 5.8 \mathrm{~mm}$ and a total length of $2 \mathrm{~m}$, resulting in an output array of $1020 \times 1020$ elements $(\sim 17.4 \mathrm{~mm} \times 17.4 \mathrm{~mm})$. This $3 \times 3$ sub-image output is depicted in Fig. 3a. A bandpass filter with a center wavelength of $600 \mathrm{~nm}$ and a FWHM of $50 \mathrm{~nm}$ is placed behind the fiber bundle output. The signals are imaged onto an intensified SCMOS camera (type: Andor iStar 18F-63) by a $60 \mathrm{~mm}$ AIS Nikon Micro objective lens (f/2.8). An image of the chessboard plate used for calibration of the optical set-up overlaid with a single shot LII-image $20 \mathrm{~ns}$ after 


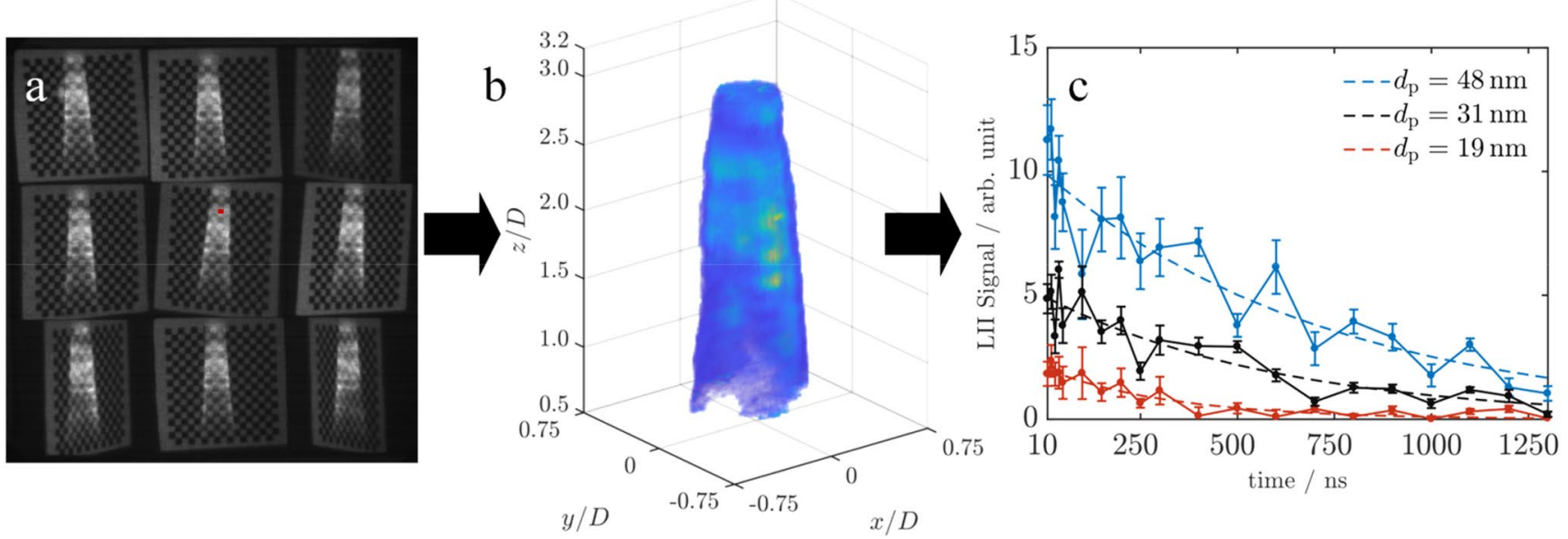

Fig. 3 Flowchart from data acquisition to LII-signals of single voxels. a An overlay of the chessboard plate used for calibration and the nine 2D images of the LII signal $20 \mathrm{~ns}$ after the laser pulse together with marked pixels chosen to calculate the SNR (red square). b The result-

the laser pulse is depicted in Fig. 3a. The camera and laser Q-switch are triggered by a pulse generator allowing for a variable delay time between the laser pulse and detection window of the LII signal while the gate time was kept constant at $10 \mathrm{~ns}$. Ten LII signals are background corrected and averaged for each of the 20 time instants, namely (10, $20,30,40,50,100,150,200,250,300,400,500,600$, $700,800,900,1000,1100,1200,1300)$ ns after the laser pulse, acquired sequentially. Even for the last time instant $(1300 \mathrm{~ns})$ the background signal from the flame luminosity was below $10 \%$ of the LII signal intensity. The shot to shot signal-to-noise ratio $(\mathrm{SNR}=\mu / \sigma)$ evaluated in a region of interest of $25 \times 25$ pixels in the center flame images (red square in Fig. 3a) is SNR $=20$ for the first time instant, decreasing for later time instances to, e.g., $\mathrm{SNR}=11$ at $500 \mathrm{~ns}$ after the laser pulse.

After the tomographic reconstruction the voxel resolution along the vertical direction is $0.25 \mathrm{~mm}$, along the other two directions it is $0.135 \mathrm{~mm}$ in a $120 \times 120 \times 120$ voxel domain. The actual spatial resolution is approximately $0.4 \mathrm{~mm} \times 0.4 \mathrm{~mm} \times 0.5 \mathrm{~mm}$ in $\mathrm{x}-, \mathrm{y}$ - and z-direction, respectively, and was evaluated from the reconstruction of a $4^{\text {th }}$ order nested cube phantom. The region covered in each sub-image is from 5 to $35 \mathrm{~mm}$ height above burner (HAB) outlet, corresponding to $z / D$-ratios from 0.46 to 3.21 . Three LII signals at a HAB of $17 \mathrm{~mm}$ and radial voxel positions of $2.5 \mathrm{~mm}$ (blue), $1.0 \mathrm{~mm}$ (black) and $0.5 \mathrm{~mm}$ (red) are depicted in Fig. 3c, showing different decay behaviors and resulting particle sizes.

As the bath gas temperature is a crucial input parameter for the LII model, a second bandpass filter with a center wavelength of $750 \mathrm{~nm}$ and a FWHM of $50 \mathrm{~nm}$ is used to compute a $3 \mathrm{D}$ ratio map for $2 \mathrm{CP}$ (gate time of camera $1 \mu \mathrm{s}$ ). ing 3D reconstruction. c Blue, black and red decay curves including the standard deviations come from the voxels at $\mathrm{HAB}=17 \mathrm{~mm}$ and radial positions of $2.5,1.0$ and $0.5 \mathrm{~mm}$, respectively. The total flame width is $6.5 \mathrm{~mm}$

Ten single shot images (without laser excitation) are averaged for both wavelength range images. The two filters were chosen to obtain a sufficient $S N R$ and decrease the effect of wavelength-dependent signal trapping. To determine the calibration constant in Eq. (5), a calibration lamp (Ocean Optics, HL-3P-CAL) is used providing a well-known radiation spectrum over a wide wavelength range.

\section{Results and discussion}

The resulting 3D temperature map from the $2 \mathrm{CP}$ measurements as well as the center slice image in the $x$-plane are depicted in Fig. 4. The observed lower temperatures in the core of the flame and the temperature increase with the radial position, followed by a rapid drop behind the flame front are in good agreement with typical temperature profiles for diffusion flames [47]. For a similar flame configuration, Yan et al. [48] reported 2D temperature maps deriving from flame images taken with a calibrated RGB-CCD camera. The overall temperature distribution is in good agreement. Yet, there are some artifacts from the reconstruction procedure and empty voxels below the signal threshold of $4 \%$ of the signal maximum (a heuristic value based on the ratio of background- to flame intensities) [33] especially at the boundaries and in the lower center region of the flame. With high probability, these high differences from pixel to pixel do not represent a physical temperature behavior and would introduce errors in the evaluation of the soot particle size. Therefore, the 3D temperature map of Fig. 4a was smoothened with a 3D median filter with a width of three voxels in each dimension. The central slice of the resulting temperature map is depicted in Fig. 4c. To account for the 

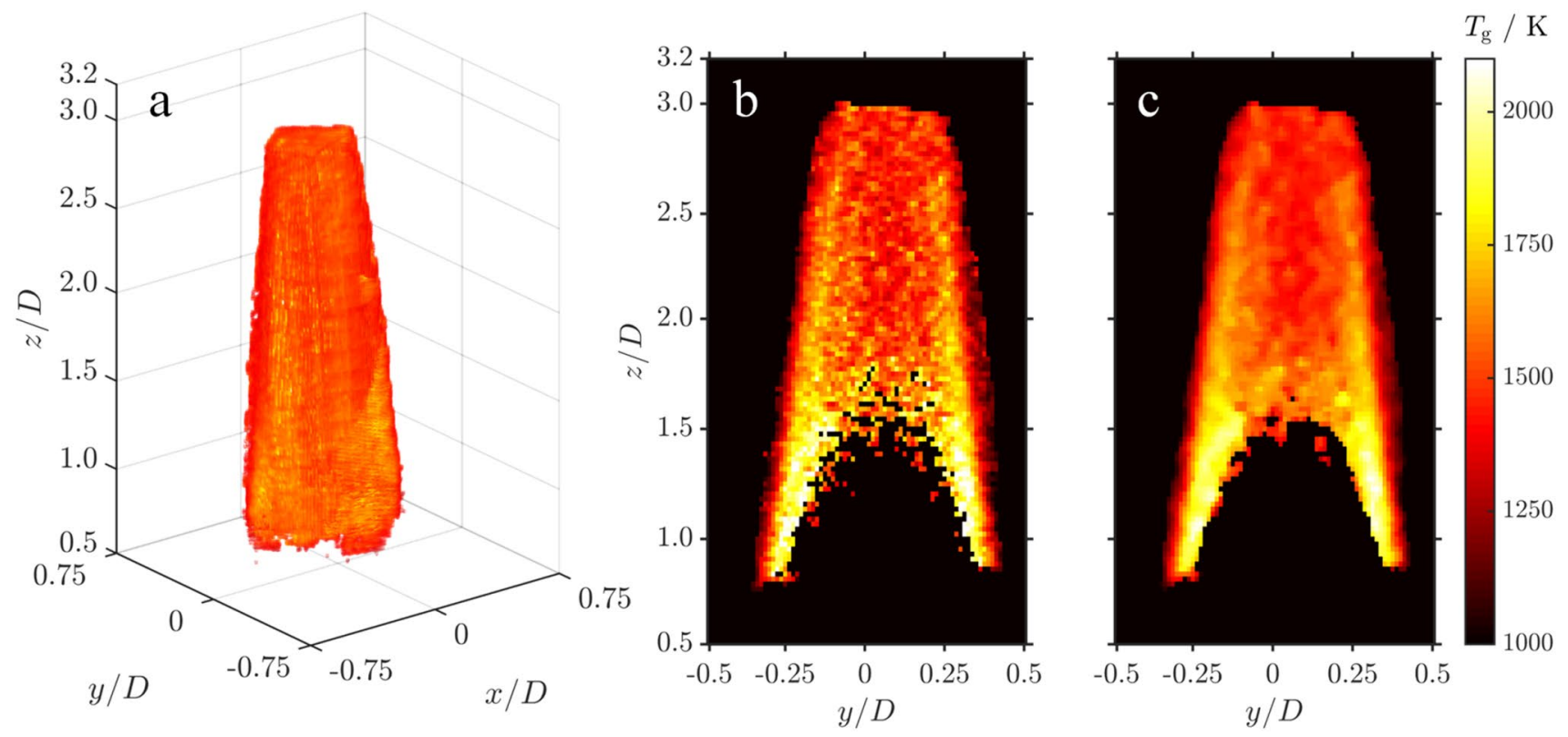

Fig. 4 a The 3D temperature map and its center slices in $x$-plane without $\mathbf{b}$ and with $\mathbf{c}$ a median filtering approach

uncertainty in $T_{\mathrm{g}}$ introduced by this filtering procedure or, e.g., the choice of optical parameters such as $E(\tilde{m})$, in the LII evaluation $T_{\mathrm{g}}$ is superimposed with a Gaussian shaped probability density with a standard deviation of $50 \mathrm{~K}$. Neglecting information about the local temperature distribution will strongly influence the inferred particle size. To demonstrate this effect, instead of using local temperatures, we also employ a homogenous temperature profile with $T_{\mathrm{g}}=1600 \mathrm{~K}$ (approximately the mean value of the temperature profile in Fig. 4a and close to temperatures chosen by previous studies as, e.g., Chen et al. [15] at the centerline position of a turbulent flame) for the LII evaluation.

To compute the spatially resolved laser fluence, the local $f_{V}$ values are used to account for the beam attenuation. As the average fluence applied in this work was below the plateau regime in the LII-fluence curve, fluctuations in the local fluence may lead to a non-linear variation in the obtained LII signal and thus $f_{V}$-value. However, as local variations in $f_{V}$ average out regarding the attenuation effect along the beam path and as the total beam attenuation is only on the order of $5-10 \%$, those local errors in $f_{V}$ lead to second order effects in the determination of $d_{\mathrm{p}}$. Based on the 3D maps for the laser fluence, the flame temperature and the LII signals at each time instant, the primary particle size within each voxel can be obtained from a regression of the signal decay with the LII model. In this work, we evaluate two scenarios:

A) The smoothed 3D temperature map (slice depicted in Fig. 4c) with an uncertainty of $50 \mathrm{~K}$ and the spatially resolved fluence map accounting for beam attenuation (average of eight laser pulses) with a relative uncertainty of $7 \%$ are used.
B) The spatially resolved laser profile is used, but the flame temperature is assumed constant over the whole flame with a value of $T_{\mathrm{g}}=1600 \mathrm{~K}$.

The resulting central slices of tomographic 3D maps of the primary particle size in the measurement volume are depicted in Fig. 5. The 3D distribution of the primary particle size of case A is depicted in Fig. 5a together with the slices through each dimension's central plane depicted on the sides of the coordinate system (the slice of the $x-y$-plane is from a z-position at $15 \mathrm{~mm} \mathrm{HAB}$ ). Figure $5 \mathrm{~b}$, c show the central $x$-plane for the cases $\mathrm{A}$ and $\mathrm{B}$, respectively, while Fig. $5 \mathrm{~d}$ is the differential image of case B minus case A. Figure 5e shows the radial distribution of Fig. $5 \mathrm{~b}, \mathrm{c}$ at $z / D=1.7$.

Obviously, there is an empty core in the center of the flame, where no soot particles are present. On the flame wings soot particles form, grow with height and start to diminish again towards the tip of the flame due to oxidation. The artifacts especially on the edges of the flame can be explained by a possible slight flame movement during the recording time of the entire signal decay curve and the $2 \mathrm{CP}$ measurements (voxels with particles larger than $80 \mathrm{~nm}$ are displayed in red color to reveal obvious artifacts). Neglecting the artifacts on the right flame side, the overall trend of particle sizes with the largest particles in the annular region of the flame is in agreement with findings of planar techniques $[14,23,49]$. While a detailed uncertainty analysis is beyond the scope of this work, it must be mentioned that due to the combination of shot-to-shot fluctuations and the reconstruction algorithm the data are noisy and therefore absolute values of particle sizes might be associated with higher uncertainties than for planar techniques. While for steady, 


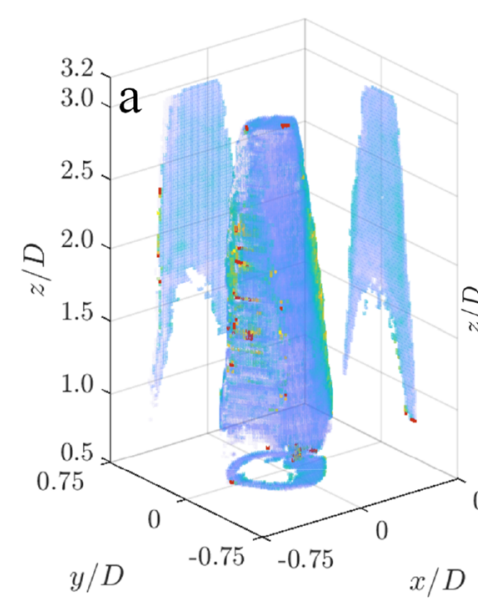

$x / D$

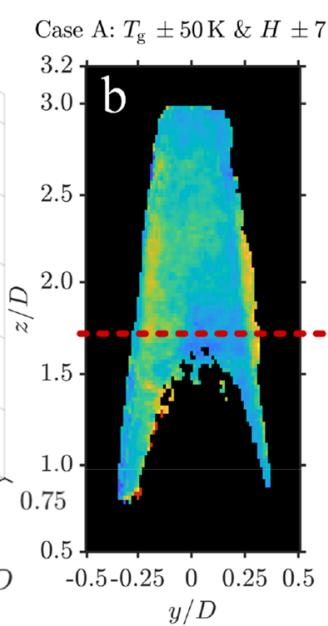

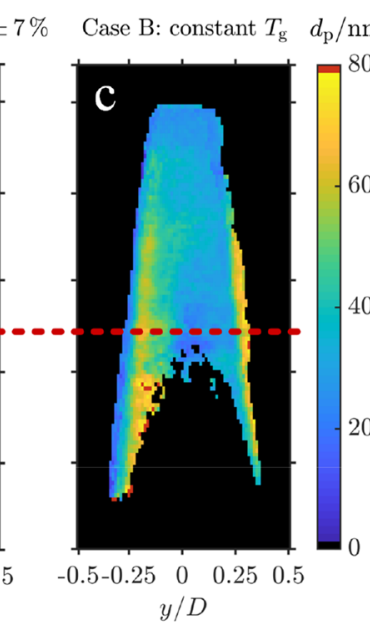
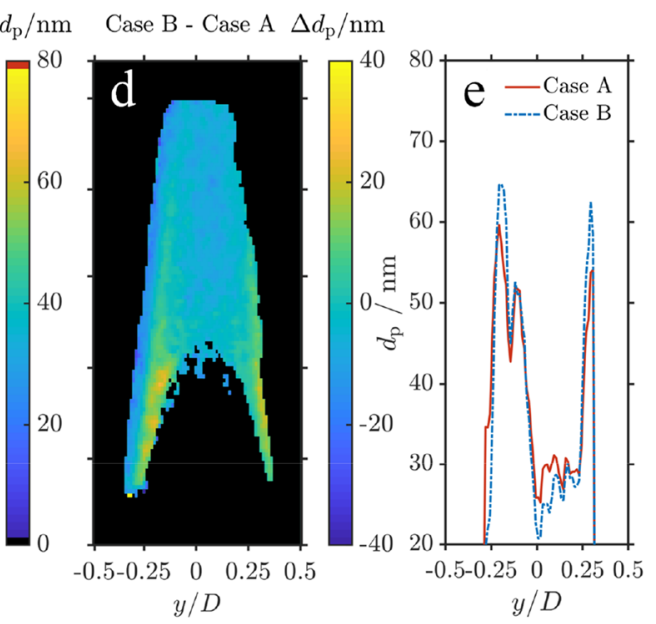

Fig. 5 a, b Show results for case A with the 3D primary particle size map and a slice through the central $\mathrm{x}-\mathrm{z}$ voxel plane. The same slice for case B is shown in c. $\mathbf{d}$ The differential image of both cases and $\mathbf{e}$

the radial distribution of the primary particle sizes (red dashed line) at $z / D=1.7$

the model predicts a faster cooling with a higher $\alpha$ value it can only be compensated by increased decay times through larger $d_{\mathrm{p}}$ values. The aggregate structure influences the accommodation coefficient as well. Considering larger aggregates at regions with larger residence times, the $\alpha$ value should decrease due to increasing effects of shielding. This countervails the effect of soot aging making the error by assuming a constant $\alpha$ less pronounced. Another effect one must be aware of and which cannot be accounted for directly because of the lack of data is that of a varying $E(\tilde{m})$ value. It is known, e.g. [29], that $E(\tilde{m})$ of incipient soot particles is lower compared to that of mature soot. Because of the presumably lower values in the regions of soot formation, $d_{\mathrm{p}}$ values are probably overestimated in these regions, yet this is problem pertinent to all LII-measurements of this kind and not specific to the tomographic approach.

\section{Conclusions}

Particle sizing by TiRe-LII was demonstrated in a threedimensional manner, forming the basis for the acquisition of soot-formation, growth and oxidation process in a more comprehensive way than with usual 2D measurements. As compared to classic tomographic approaches, the expenditure on camera equipment was drastically reduced by using a customized fiber bundle, allowing to acquire nine images from different angels with one camera at the same time. A steady ethene diffusion flame was used for a first demonstration of the approach, of course not fully exploiting its capabilities. Yet, the analysis has also shown that with the assumption of an average temperature or laser fluence even the overall picture of soot size evolution may be completely 
faulty. While the fluence profile of the laser may be obtained in a single calibration measurement, a simultaneous acquisition of the 3D temperature distribution is thus essential for application in a non-stationary flame. While an approach with two cameras each for TiRe-LII and 2CP measurements is straightforward, the task might also be accomplished with only three cameras for high-speed measurements where high-speed equipment is required. To this end one highspeed laser and three high-speed cameras running in the $\mathrm{kHz}$ range would be needed: two cameras equipped with the same bandpass filters and delayed by a few hundred nanoseconds for the detection of the LII-signal decay after the laser pulse. A third camera, equipped with another bandpass filter, could be synchronized with one of the other cameras to accomplish 2CP measurements in between the laser pulses. This approach would require an additional beam splitter, switching of gate times between LII and 2CP measurements and possibly a temporal interpolation of the temperature field for the LII evaluation if the temperature measurement is not executed very shortly before the laser pulse.

However, thanks to existing tomographic approaches employing fiber bundles, this technique for 3D high-speed particle sizing would still come at manageable experimental cost.

Acknowledgements We gratefully acknowledge support by the Erlangen Graduate School in Advanced Optical Technologies (SAOT) and the China Scholarship Council, CSC (research stay of T. Yu).

Funding Open Access funding enabled and organized by Projekt DEAL.

Open Access This article is licensed under a Creative Commons Attribution 4.0 International License, which permits use, sharing, adaptation, distribution and reproduction in any medium or format, as long as you give appropriate credit to the original author(s) and the source, provide a link to the Creative Commons licence, and indicate if changes were made. The images or other third party material in this article are included in the article's Creative Commons licence, unless indicated otherwise in a credit line to the material. If material is not included in the article's Creative Commons licence and your intended use is not permitted by statutory regulation or exceeds the permitted use, you will need to obtain permission directly from the copyright holder. To view a copy of this licence, visit http://creativecommons.org/licenses/by/4.0/.

\section{References}

1. H. Wang, Proc. Combust. Inst. 33, 41-67 (2011)

2. H. Bockhorn, Soot Formation in Combustion: Mechanisms and Models (Springer, New York, 2013).

3. H. Michelsen, Proc. Combust. Inst. 36, 717-735 (2017)

4. H. Michelsen, C. Schulz, G. Smallwood, S. Will, Prog. Energy Combust. Sci. 51, 2-48 (2015)

5. E. Cenker, G. Bruneaux, T. Dreier, C. Schulz, Appl. Phys. B 119, 745-763 (2015)

6. F. Liu, D. Snelling, K. Thomson, G. Smallwood, Appl. Phys. B 96, 623-636 (2009)
7. F.J. Bauer, K.J. Daun, F.J.T. Huber, S. Will, Appl. Phys. B 125, 109 (2019)

8. B. Crosland, K. Thomson, M. Johnson, Appl. Phys. B 112, 381$393(2013)$

9. H. Bladh, J. Johnsson, P.-E. Bengtsson, Appl. Phys. B 90, 109-125 (2008)

10. B. Quay, T.-W. Lee, T. Ni, R. Santoro, Combust. Flame 97, 384 392 (1994)

11. S. Will, S. Schraml, A. Leipertz, Opt. Lett. 20, 2342-2344 (1995)

12. Z. Sun, D. Gu, G. Nathan, Z. Alwahabi, B. Dally, Proc. Combust. Inst. 35, 3673-3680 (2015)

13. D. Gu, Z. Sun, B.B. Dally, P.R. Medwell, Z.T. Alwahabi, G.J. Nathan, Combust. Flame 179, 33-50 (2017)

14. R. Hadef, K.P. Geigle, J. Zerbs, R.A. Sawchuk, D.R. Snelling, Appl. Phys. B 112, 395-408 (2013)

15. Y. Chen, E. Cenker, D.R. Richardson, S.P. Kearney, B.R. Halls, S.A. Skeen, C.R. Shaddix, D.R. Guildenbecher, Opt. Lett. 43, 5363-5366 (2018)

16. J.B. Michael, P. Venkateswaran, C.R. Shaddix, T.R. Meyer, Appl. Opt. 54, 3331-3344 (2015)

17. J. Hult, A. Omrane, J. Nygren, C. Kaminski, B. Axelsson, R. Collin, P.-E. Bengtsson, M. Aldén, Exp. Fluids 33, 265-269 (2002)

18. T.R. Meyer, B.R. Halls, N. Jiang, M.N. Slipchenko, S. Roy, J.R. Gord, Opt. Express 24, 29547-29555 (2016)

19. E.M. Hall, B.R. Halls, D.R. Richardson, D.R. Guildenbecher, E. Cenker, M. Paciaroni, in AIAA Scitech 2020 Forum, 2020, pp. 2209

20. N. Anikin, R. Suntz, H. Bockhorn, Appl. Phys. B 107, 591-602 (2012)

21. M. Kang, X. Li, L. Ma, Proc. Combust. Inst. 35, 3821-3828 (2015)

22. H. Liu, B. Sun, W. Cai, Opt. Commun. 437, 33-43 (2019)

23. B. Tian, C. Zhang, Y. Gao, S. Hochgreb, Aerosol Sci. Tech. 51, 1345-1353 (2017)

24. R. Mansmann, T.A. Sipkens, J. Menser, K.J. Daun, T. Dreier, C. Schulz, Appl. Phys. B 125, 126 (2019)

25. S.A. Kuhlmann, J. Reimann, S. Will, Chem. Ing. Tech. 81, 803809 (2009)

26. A. Filippov, M. Zurita, D. Rosner, J. Colloid Interface Sci. 229, 261-273 (2000)

27. S. Maffi, S. De Iuliis, F. Cignoli, G. Zizak, Appl. Phys. B 104, 357-366 (2011)

28. D.R. Snelling, F. Liu, G.J. Smallwood, Ö.L. Gülder, Combust. Flame 136, 180-190 (2004)

29. N.-E. Olofsson, J. Simonsson, S. Török, H. Bladh, P.-E. Bengtsson, Appl. Phys. B 119, 669-683 (2015)

30. P.J. Hadwin, T. Sipkens, K. Thomson, F. Liu, K. Daun, Appl. Phys. B 122, 1 (2016)

31. R. Puri, T. Richardson, R. Santoro, R. Dobbins, Combust. Flame 92, 320-333 (1993)

32. M.W. Chase Jr., J. Phys. Chem. Ref. Data 14, 535 (1998)

33. T. Yu, F.J. Bauer, F.J.T. Huber, S. Will, W. Cai: Opt. Exp. (2020, submitted)

34. J. Yon, R. Lemaire, E. Therssen, P. Desgroux, A. Coppalle, K. Ren, Appl. Phys. B 104, 253-271 (2011)

35. J.-Y. Bouguet: (2008) http://www.vision.caltech.edu/bouguetj/ calib_doc/

36. K. Mohri, S. Görs, J. Schöler, A. Rittler, T. Dreier, C. Schulz, A. Kempf, Appl. Opt. 56, 7385-7395 (2017)

37. T. Yu, C. Ruan, H. Liu, W. Cai, X. Lu, Appl. Opt. 57, 5962-5969 (2018)

38. N. Denisova, P. Tretyakov, A. Tupikin, Combust. Flame 160, 577-588 (2013)

39. S.J. Grauer, P.J. Hadwin, K.J. Daun, Appl. Opt. 55, 5772-5782 (2016) 
40. K.J. Daun, S.J. Grauer, P.J. Hadwin, J. Quant. Spectrosc. Radiat. Transf. 172, 58-74 (2016)

41. F.S. Prol, P.O. Camargo, M.T. Muella, Boletim. Ciências. Geodés 89, 1531-1542 (2017)

42. P. Subbarao, P. Munshi, K. Muralidhar, Numer. Heat Transf 31, 347-372 (1997)

43. J. Floyd, P. Geipel, A. Kempf, Combust. Flame 158, 376-391 (2011)

44. C. Sorensen, Aerosol Sci. Technol. 35, 648-687 (2001)

45. D.R. Snelling, K.A. Thomson, G.J. Smallwood, Ö.L. Gülder, Appl. Opt. 38, 2478-2485 (1999)

46. C. Schulz, B.F. Kock, M. Hofmann, H. Michelsen, S. Will, B. Bougie, R. Suntz, G. Smallwood, Appl. Phys. B 83, 333 (2006)
47. J. Cruz, L.F. Figueira da Silva, F. Escudero, F. Cepeda, J. ElicerCortés, A. Fuentes: Combust. Sci. Technol. 1-18 (2020)

48. W. Yan, D. Chen, Z. Yang, E. Yan, P. Zhao, Energies 10, 750 (2017)

49. S. Will, S. Schraml, A. Leipertz, Symp. Combust. Proc. 26, $2277-$ 2284 (1996)

Publisher's Note Springer Nature remains neutral with regard to jurisdictional claims in published maps and institutional affiliations. 